\title{
Block-Diagonalization Problem for the Common Hückel-Type Hamiltonian Matrix of Alternant Hydrocarbons and Its Nonperturbative Solution
}

\author{
V. GINEITYTE \\ Institute of Theoretical Physics and Astronomy, Vilnius University, Gostauto 12, \\ 2600 Vilnius, Lithuania \\ Received 9 January 2004; accepted 22 April 2004 \\ Published online 6 October 2004 in Wiley InterScience (www.interscience.wiley.com). \\ DOI 10.1002/qua.20215
}

\begin{abstract}
The block-diagonalization problem originating from the Brillouin theorem and determining the noncanonical molecular orbitals (NCMOs) has been formulated and solved for the common Hamiltonian matrix $\mathbf{H}$ of alternant hydrocarbons in the framework of the simple Hückel model. The well-known way of partitioning of the total basis set of $2 p_{z}$ AOs of carbon atoms of these molecules into two subsets allowed the problem to be solved nonperturbatively in terms of entire subsets of atomic orbitals (AOs), and thereby of respective submatrices (blocks) of the matrix $\mathbf{H}$, without specifying either the internal structures or dimensions of the latter. As a result, the NCMOs of alternant hydrocarbons proved to be characterized by common peculiarities, including uniform total extents of delocalization. A close relationship has been established between the block-diagonalization problem under study and the commutation equation determining the respective one-electron density matrix (DM) directly without invoking the canonical molecular orbitals (CMOs). In particular, the off-diagonal blocks both of the NCMO representation matrix $\mathrm{C}$ and of the DM $\mathbf{P}$ were shown to follow from the same matrix condition originally suggested by G. G. Hall on the basis of structures of CMOs of alternant hydrocarbons. The relationship between the two fundamental noncanonical problems gave an additional insight into the origin of the observed similarity between constitutions of matrices $\mathbf{C}$ and $\mathbf{P}$. It has been concluded on this basis that the common DM of alternant hydrocarbons (the charge-bond order matrix) is among noncanonical (localized) ways of describing electronic structures whatever the actual method of its derivation. (c) 2004 Wiley Periodicals, Inc. Int J Quantum Chem 101: 274-282, 2005
\end{abstract}

Key words: Brillouin theorem; noncanonical MOs; one-electron density matrix; alternant hydrocarbons; block-diagonalization problem 


\section{Introduction}

$\mathbf{T}$ he most popular standard method of molecular orbitals (MOs) is based on solution of the canonical Hartree-Fock (HF) equation [1-3], accordingly called the canonical molecular orbital (CMO) method. Owing to the relation of eigenvalues of the Fockian matrix to ionization potentials of molecule (cf. the Koopmans theorem [4]), canonical MOs may be regarded as the most appropriate orbitals for representing one-electron energies, and thereby the spectral properties of molecular systems.

Instead of energy levels, however, the supposed different extents of localization of electrons in the real space form the basis of the chemical classification of molecules. For example, three principal classes of hydrocarbons are usually distinguished [5-7], viz. the saturated hydrocarbons, their unsaturated aliphatic analogues, and the aromatic hydrocarbons; these are assumed to be characterized by increasing extents of delocalization of electron pairs pertinent to individual chemical bonds. As far as the CMOs are concerned, these orbitals usually embrace the whole molecule under study whatever its chemical structure (see e.g., Ref. [8]) and, consequently, exhibit no substantial differences for different classes of compounds. That is why CMOs are hardly the optimum orbitals for reflecting chemical structures and their alterations when passing from one type of compound to another. In this connection, the development of alternative approaches to the CMO method becomes important.

In contrast to the unique canonical HF equation, various forms of the noncanonical one-electron problem are possible [1, 3]. In our context, the direct way of obtaining the noncanonical molecular orbitals (NCMOs) [9-17] on the basis of the Brillouin theorem $[3,18]$ deserves particular attention. The point is that no "external" localization criteria are invoked in this approach, and thereby the actual extents of localization of NCMOs may be expected to depend only on the "internal" factors, including the chemical constitution of the given compound.

Among particular forms of the Brillouin theorem, there is a zero value requirement for an offdiagonal element of the Fockian operator referring to an occupied $\mathrm{MO}$ and a vacant $\mathrm{MO}$ [3]. In its matrix form, this requirement resolves itself into the zero matrix condition for the occupied-vacant off-diagonal block (submatrix) of the total Fockian matrix in the basis of NCMOs being sought [10-16].
As a result, the block-diagonalization problem for the Fockian matrix is obtained.

As compared with the more popular diagonalization problem that results from the canonical HF equation, methods of solution of the block-diagonalization problem are considerably less developed. That is why the actual search for NCMOs on the basis of the Brillouin theorem [9-17] was restricted to applications of various forms of the perturbation theory. Nevertheless, the principal achievements of these contributions (enumerated below) are promising for further developments in this field. First, conditions that ensure the existence of NCMOs of the desired type (viz. of NCMOs of the bond-orbital-and-tail structure) have been explored [10]. Second, general solutions of the blockdiagonalization problem have been obtained in terms of entire submatrices (blocks) of the initial matrix without specifying either the internal constitution or the dimensions of these submatrices [1317]. (Solutions of this type refer to definite classes of initial matrices, and thereby of molecules.) The third, and most important, achievement, however, consists of the establishment of a close relationship between perturbative solutions of the block-diagonalization problem and of the noncanonical problem that determines the respective one-electron density matrix (DM) [13-15], and thereby between the resulting NCMO representation matrix and the unique DM of the given molecule. The latter may be obtained either indirectly by summing up coefficients of occupied CMOs or directly on the basis of solution of the so-called commutation equation [19].

The one-electron DM of the molecule (the charge-bond order matrix) is among the most fundamental quantum chemical characteristics related to numerous observed properties [1, 2]. This ensures an increased significance of NCMOs related to the DM, as closely as possible. Again, the abovementioned principal relationship between the NCMO representation matrix and the DM allowed the latter to be considered as a part of the noncanonical (localized) description of electronic structures [15].

In this context, an important question arises about the validity of the above-enumerated conclusions beyond the scope of applicability of perturbative methods. To be able to explore this point, nonperturbative solutions of the block-diagonalization problem are evidently required; expressing them in terms of entire submatrices of the initial matrix also 
is highly desirable. This study is devoted to the development of methods of just this type.

We will consider the so-called alternant hydrocarbons $[3,19-25]$ that embrace most aromatic hydrocarbons characterized by the divergence of perturbative solutions of the block-diagonalization problem [20]. Within the framework of the simple Hückel model, these systems may be represented by a common model Hamiltonian matrix $\mathbf{H}$ consisting of four blocks (submatrices) and reflecting the relevant chemical structures directly [23]. Moreover, the existence of expressions for the chargebond order matrix of alternant hydrocarbons in terms of entire blocks of the matrix $\mathbf{H}[23,24]$ gives us a hint that an analogous form of the NCMO representation matrix is also feasible. These circumstances allowed us to formulate the respective common block-diagonalization problem and to suggest its general solution (Section 2). Thereupon, we will demonstrate the feasibility of a related solution of the commutation equation for the charge-bond order matrix (Section 3). Finally, we turn to a discussion of the results obtained (Section 4).

\section{Solution of the Block-Diagonalization Problem}

Let us begin with the most common case of even alternant hydrocarbons containing $2 n$ carbon atoms. The basis set $\{\chi\}$ of such a system consists of $2 n$ $2 p_{z}$ AOs of these atoms, and may always be divided into two $n$-dimensional subsets so that the intrasubset resonance parameters take zero values [20, 23, 24]. It happens in this way because the neighboring pairs of AOs characterized by nonzero resonance parameters in the Hückel model get into different subsets. Moreover, the Coulomb parameters representing AOs usually are assumed to take uniform values $\alpha$, and the equality $\alpha=0$ is accepted for convenience.

As a result, the one-electron Hamiltonian matrices of our systems acquire a common form containing zero submatrices (blocks) in its diagonal positions:

$$
\mathbf{H}=\left|\begin{array}{cc}
\mathbf{0} & \mathbf{B} \\
\mathbf{B}^{+} & \mathbf{0}
\end{array}\right| .
$$

Again, $\mathbf{B}$ and $\mathbf{B}^{+}$are $n \times n$-dimensional off-diagonal blocks containing intersubset resonance parameters. Nonzero elements of these blocks represent the neighboring pairs of $2 p_{z}$ AOs. The mean value of the latter $\beta$ will be used here as a (negative) energy unit by accepting the equality $\beta=1$. The superscript + of Eq. (1) designates the transposed matrix $\mathbf{B}$. Note that $\mathbf{B} \neq \mathbf{B}^{+}$in the general case.

In accordance with the Brillouin theorem [1017], turning from the above-specified basis of AOs $\{\chi\}$ to the set of NCMOs being sought $\{\varphi\}$ may be carried out by transforming the initial matrix $\mathbf{H}$ of Eq. (1) into the following block-diagonal form:

$$
\mathbf{H}^{\prime}=\mathbf{C}^{-1} \mathbf{H C}=\left|\begin{array}{cc}
\mathbf{E}_{1} & \mathbf{0} \\
\mathbf{0} & \mathbf{E}_{2}
\end{array}\right|,
$$

where $\mathbf{C}$ is the transformation matrix, and $\mathbf{E}_{1}$ and $\mathbf{E}_{2}$ are $n \times n$-dimensional submatrices (blocks) that may be referred to as eigenblocks of the matrix $\mathbf{H}$ [14]. As in Refs. [13-17], let us also impose a unitarity requirement for the matrix $\mathbf{C}$, i.e.,

$$
\mathbf{C}^{+} \mathbf{C}=\mathbf{I}_{(2 n)},
$$

where $\mathbf{I}_{(2 n)}$ stands for a $2 n$-dimensional unit matrix.

Let us look for the solution of our problem of the following constitution:

$$
\mathbf{C}=\frac{1}{\sqrt{2}}\left|\begin{array}{cc}
\mathbf{I} & \mathbf{Z} \\
\mathbf{X} & -\mathbf{I}
\end{array}\right|,
$$

where I coincides with an $n$-dimensional unit matrix and $\mathbf{X}$ and $\mathbf{Z}$ are $n \times n$-dimensional submatrices to be found. Equation (4) and the equality $\mathrm{C}^{-1}=\mathrm{C}^{+}$ resulting from Eq. (3) should then be substituted into zero-matrix conditions for off-diagonal blocks of the product $\mathrm{C}^{-1} \mathrm{HC}$. As a result, we obtain the following requirement

$$
\mathbf{B}=\mathbf{X}^{+} \mathbf{B}^{+} \mathbf{Z}
$$

and its transposed counterpart. Again, Eq. (3) yields

$$
\mathbf{X}^{+} \mathbf{X}=\mathbf{I}, \quad \mathbf{Z}^{+} \mathbf{Z}=\mathbf{I}, \quad \mathbf{X}=\mathbf{Z}^{+} .
$$

The coincidence of matrices $\mathbf{X}$ and $\mathbf{Z}^{+}$shown in Eq. (6) implies that the matrix $\mathbf{C}$ is both unitary and Hermitian. Using the last relation of Eq. (6) within Eq. (5), the latter may be rewritten in the form

$$
\mathbf{B}=\mathbf{Z B}^{+} \mathbf{Z}
$$

This square matrix problem, in turn, may be reformulated as a symmetry requirement for the 
matrix either $\mathbf{B Z}^{+}$or $\mathbf{B}^{+} \mathbf{Z}$, provided that the unitarity condition for the matrix $\mathbf{Z}$ shown in Eq. (6) is also used. The above-expected alternative requirements are

$$
\begin{aligned}
& \mathbf{B Z}^{+}=\left(\mathbf{B Z}^{+}\right)^{+}=\mathbf{Z B}^{+} \\
& \mathbf{B}^{+} \mathbf{Z}=\left(\mathbf{B}^{+} \mathbf{Z}\right)^{+}=\mathbf{Z}^{+} \mathbf{B}
\end{aligned}
$$

and coincide with those used in Ref. [24] when obtaining the one-electron DM of alternant hydrocarbons. In this connection, let us call the relations of Eqs. (8) and (9) the Hall conditions. The expressions for the matrix $\mathbf{Z}$ in terms of matrices $\mathbf{B}$ and $\mathbf{B}^{+}$ may also be found as described in Ref. [24]. Instead, we will invoke here the concept of the left and right polar decomposition of matrices [25, 26]. To this end, let us introduce the notations

$$
\mathbf{B Z}^{+}=\mathbf{S}, \quad \mathbf{Z}^{+} \mathbf{B}=\mathbf{T},
$$

where $\mathbf{S}$ and $\mathbf{T}$ are Hermitian matrices, as indicated in Eqs. (8) and (9). These relations may be multiplied by the matrix $\mathbf{Z}$ from their right- and left-hand sides, respectively, and the unitarity condition for $\mathbf{Z}$ shown in Eq. (6) may be used. The result then takes the form of two alternative expressions for the matrix B, viz.:

$$
\mathbf{B}=\mathbf{S Z}, \quad \mathbf{B}=\mathbf{Z T} .
$$

These relations correspondingly represent the left and right polar decompositions of the matrix $\mathbf{B}$, where $\mathbf{S}$ and $\mathbf{T}$ are the respective unique Hermitian components, defined as follows:

$$
\mathbf{S}=\left(\mathbf{B B}^{+}\right)^{1 / 2}, \quad \mathbf{T}=\left(\mathbf{B}^{+} \mathbf{B}\right)^{1 / 2}
$$

(see Theorem 7.3.2 and Corollary 7.3.3 of Ref. [25]). Given that B is a nonsingular matrix, the common unitary component of these decompositions, i.e., matrix $\mathbf{Z}$, is also a unique matrix, which may be correspondingly expressed in the form

$$
\mathbf{Z}=\mathbf{R B}, \quad \mathbf{Z}=\mathbf{B Q},
$$

where

$$
\mathbf{R}=\left(\mathbf{B B}^{+}\right)^{-1 / 2}, \quad \mathbf{Q}=\left(\mathbf{B}^{+} \mathbf{B}\right)^{-1 / 2}
$$

and

$$
\mathbf{R} \mathbf{B}=\mathbf{B Q} .
$$

It is also noteworthy that the products $\mathbf{B B}^{+}$and $\mathbf{B}^{+} \mathbf{B}$ are positive-definite matrices under the same condition of nonsingularity of matrix B (cf. Theorem 7.2.7 of Ref. [25]).

As compared with the solution of the matrix problem of Eqs. (8) and (9) suggested in Ref. [24], the above-outlined alternative is based on more general results of the matrix algebra.

Using Eqs. (4), (6), and (13)-(15), the NCMO representation matrix $\mathbf{C}$ may be expressed in two ways, viz.:

$$
\mathbf{C}=\frac{1}{\sqrt{2}}\left|\begin{array}{cr}
\mathbf{I} & \mathbf{R B} \\
\mathbf{B}^{+} \mathbf{R} & -\mathbf{I}
\end{array}\right|, \quad \mathbf{C}=\frac{1}{\sqrt{2}}\left|\begin{array}{cr}
\mathbf{I} & \mathbf{B Q} \\
\mathbf{Q B}^{+} & -\mathbf{I}
\end{array}\right| .
$$

The expressions for eigenblocks $\mathbf{E}_{1}$ and $\mathbf{E}_{2}$ may be derived using the diagonal blocks of the product $\mathbf{C}^{-1} \mathbf{H C}$. We then obtain

$$
\mathbf{E}_{1}=\frac{1}{2}\left[\mathbf{X}^{+} \mathbf{B}^{+}+\mathbf{B} \mathbf{X}\right], \quad \mathbf{E}_{2}=-\frac{1}{2}\left[\mathbf{Z}^{+} \mathbf{B}+\mathbf{B}^{+} \mathbf{Z}\right] .
$$

The first expression of Eq. (17) may easily be reformulated in terms of $\mathbf{Z}$ and $\mathbf{Z}^{+}$, after substituting the last relation of Eq. (6). Comparison of the final formulae for $\mathbf{E}_{1}$ and $\mathbf{E}_{2}$ with those for $\mathbf{S}, \mathbf{S}^{+}, \mathbf{T}$, and $\mathbf{T}^{+}$shown in Eqs. (8), (9), and (10) indicates that the eigenblocks of the Hamiltonian matrix of even alternant hydrocarbons are directly related to the Hermitian components of the off-diagonal blocks B, i.e.,

$$
\mathbf{E}_{1}=\mathbf{S}=\left(\mathbf{B B}^{+}\right)^{1 / 2}, \quad \mathbf{E}_{2}=-\mathbf{T}=-\left(\mathbf{B}^{+} \mathbf{B}\right)^{1 / 2} .
$$

Squares of the eigenblocks in Eq. (18) coincide with matrices $\mathbf{B B}^{+}$and $\mathbf{B}^{+} \mathbf{B}$ obtained in Ref. [27], when reformulating the eigenvalue problem for the $2 n \times 2 n$-dimensional matrix $\mathbf{H}$ of Eq. (1) into two $n \times n$-dimensional problems. It should also be noted that unique positive-definite square roots may always be defined for Hermitian positivedefinite matrices $\mathbf{B B}^{+}$and $\mathbf{B}^{+} \mathbf{B}$ [25].

No zero eigenvalues are known to arise in the spectra of Hamiltonian matrices of even alternant hydrocarbons of a finite size [24]. (The unusual systems containing isolated $2 p_{z}$ AOs are excluded here.) Consequently, an assumption that the products $\mathbf{B B}^{+}$and $\mathbf{B}^{+} \mathbf{B}$ are positive-definite matrices appears appropriate for these systems. Given that this is the case, the spectra of the relevant eigen- 
blocks $E_{1}$ and $E_{2}$ do not overlap one with another in addition.

Let us define now two $n$-dimensional row-matrices $\left(\chi_{1}\right)$ and $\left(\chi_{2}\right)$ containing the subsets of AOs underlying the blocks of the initial matrix $\mathbf{H}$ of Eq. (1). The relevant row-matrices $\left(\varphi_{(+)}\right)$and $\left(\varphi_{(-)}\right)$will consist of the occupied and vacant NCMOs, respectively. Using Eq. (16), the latter may be expressed as follows:

$$
\begin{aligned}
\left(\varphi_{(+)}\right)=\frac{1}{\sqrt{2}}\left[\left(\chi_{1}\right)+\right. & \left.\left(\chi_{2}\right) \mathbf{B}^{+} \mathbf{R}\right] \\
\left(\varphi_{(-)}\right) & =\frac{1}{\sqrt{2}}\left[\left(\chi_{1}\right) \mathbf{R B}-\left(\chi_{2}\right)\right],
\end{aligned}
$$

where the $\mathbf{R}$ - representation of the matrix $\mathbf{C}$ is used. The occupied NCMO $\varphi_{(+) i}$ may be given here as an example

$$
\boldsymbol{\varphi}_{(+) i}=\frac{1}{\sqrt{2}}\left[\chi_{1, i}+\sum_{k}^{(2)} \chi_{2, k}\left(\mathbf{B}^{+} \mathbf{R}\right)_{k i}\right],
$$

where the sum over $k$ embraces AOs of the second subset and

$$
\sum_{k}^{(2)}\left[\left(\mathbf{B}^{+} \mathbf{R}\right)_{k i}\right]^{2}=1
$$

Thus, the noncanonical MOs proposed in Refs. $[11,12]$ acquire a very natural form shown in Eq. (20) in the case of even alternant hydrocarbons. The relation of Eq. (21) follows from the unitarity condition for the matrix $\mathbf{Z}=\mathbf{R} \mathbf{B}$ [cf. Eqs. (6) and (13)] and represents the normalization condition for NCMOs.

As shown in Eqs. (19) and (20), the occupied and vacant NCMOs are attached to subsets $\left\{\chi_{1}\right\}$ and $\left\{\chi_{2}\right\}$, respectively. Equivalence of both subsets of AOs allows us, then, to expect that an alternative solution of our problem is feasible, wherein the subsets $\left\{\chi_{1}\right\}$ and $\left\{\chi_{2}\right\}$ are interchanged.

To make sure that it is the case, no more is required than to choose the initial solution of the form

$$
\mathbf{C}^{\prime}=\frac{1}{\sqrt{2}}\left|\begin{array}{cc}
-\mathbf{I} & \mathbf{Z} \\
\mathbf{X} & \mathbf{I}
\end{array}\right|
$$

instead of Eq. (4). Substituting this matrix into Eqs. (2) and (3) yields the same principal relations for the matrix $\mathbf{Z}$ shown in Eqs. (5)-(9). As a result, the derivation represented by Eqs. (10)-(15) is also valid, and we obtain

$$
\mathbf{C}^{\prime}=\frac{1}{\sqrt{2}}\left|\begin{array}{cc}
-\mathbf{I} & \mathbf{R B} \\
\mathbf{B}^{+} \mathbf{R} & \mathbf{I}
\end{array}\right|, \quad \mathbf{C}^{\prime}=\frac{1}{\sqrt{2}}\left|\begin{array}{cc}
-\mathbf{I} & \mathbf{B Q} \\
\mathbf{Q B}^{+} & \mathbf{I}
\end{array}\right|
$$

and

$$
\begin{array}{r}
\left(\varphi_{(+)}^{\prime}\right)=\frac{1}{\sqrt{2}}\left[\left(\chi_{1}\right) \mathbf{R} \mathbf{B}+\left(\chi_{2}\right)\right], \quad\left(\varphi_{(-)}^{\prime}\right)=\frac{1}{\sqrt{2}}\left[-\left(\chi_{1}\right)\right. \\
\left.+\left(\chi_{2}\right) \mathbf{B}^{+} \mathbf{R}\right]
\end{array}
$$

instead of Eqs. (16) and (19), respectively. It is seen that the occupied NCMOs prove to be attached to the second subset $\left\{\chi_{2}\right\}$ in this case, e.g.,

$$
\varphi_{(+) p}^{\prime}=\frac{1}{\sqrt{2}}\left[\chi_{2, p}+\sum_{q}^{(1)} \chi_{1, q}(\mathbf{R B})_{q p}\right] .
$$

Finally, the stabilization energy of our system may be found on the basis of Eq. (18) and takes the form

$$
\Delta E_{\text {stab }}=2 \operatorname{Trace}_{1}=2 \operatorname{Trace}\left[\left(\mathbf{B B}^{+}\right)^{1 / 2}\right] .
$$

Before finishing this section, let us consider the feasibility of extending the above results to ions of odd alternant hydrocarbons involving an even total number of $\pi$-electrons ( $2 n)$. For cations of these hydrocarbons characterized by $2 n+1 \mathrm{AO}$, the first and second subset will contain $n$ and $n+1$ basis functions, respectively. As a result, blocks $\mathbf{B}$ and $\mathbf{Z}$ become $n \times(n+1)$-dimensional matrices, whereas $\mathbf{B Z}^{+}, \mathbf{B}^{+} \mathbf{Z}, \mathbf{B B}^{+}, \mathbf{R}$, and $\mathbf{Q}$ remain square matrices of corresponding dimensions. This implies that the $n \times n$-dimensional eigenblock $\mathbf{E}_{1}$ and the $(n+1) \times$ $(n+1)$-dimensional block $\mathbf{E}_{2}$ refer to occupied and vacant NCMOs, respectively. In the case of anions, the subset of the higher dimension will be attributed accordingly to occupied NCMOs. However, the presence of a zero eigenvalue in the spectra of odd alternant hydrocarbons [21, 22] requires that we confine ourselves to the $n \times n$-dimensional products, viz. to $\mathbf{B B}^{+}$and $\mathbf{B}^{+} \mathbf{B}$ for cations and anions, respectively. Correspondingly, this actually implies confinements to definitions of only $\mathbf{R}-$ and $\mathrm{Q}-$ representations of NCMOs. 


\section{Relation Between the Block-Diagonalization Problem and the Commutation Equation for the One-Electron DM}

As was mentioned, the charge-bond order matrix of alternant hydrocarbons $\mathbf{P}$ has been expressed in Ref. [24] in terms of entire blocks $\mathbf{B}$ and $\mathbf{B}^{+}$, using the symmetry requirements for matrices $\mathbf{B Z}^{+}$and $\mathbf{B}^{+} \mathbf{Z}$ of Eqs. (8) and (9). It is no surprise, in this connection, that the final expressions for the matrix $\mathbf{P}$ obtained there, viz.:

$$
\mathbf{P}=\left|\begin{array}{cc}
\mathbf{I} & \mathbf{R B} \\
\mathbf{B}^{+} \mathbf{R} & \mathbf{I}
\end{array}\right|, \quad \mathbf{P}=\left|\begin{array}{cc}
\mathbf{I} & \mathbf{B Q} \\
\mathbf{Q B}^{+} & \mathbf{I}
\end{array}\right|
$$

are highly similar to those representing the NCMOs and shown in Eqs. (16) and (23).

The Hall conditions of Eqs. (8) and (9) were originally derived [24] by invoking the structures of CMOs of alternant hydrocarbons. Our aim consists of an independent derivation of the same conditions without any reference to CMOs.

To this end, let us begin with the fundamental problem for the DM, consisting of three equations $[15,19]$, viz.:

$$
\begin{gathered}
{[\mathbf{H}, \mathbf{Y}]_{-}=\mathbf{0},} \\
\mathbf{Y}^{2}=\mathbf{I},
\end{gathered}
$$

$$
\text { Trace } \mathbf{Y}=0 \text {, }
$$

where $\mathbf{Y}$ stands for the so-called residual charge matrix connected to $\mathbf{P}$ by the relation $\mathbf{Y}=\mathbf{P}-\mathbf{I}$ and the notation $[\ldots, . .]_{-}$indicates a commutator of matrices.

The commutation condition of Eq. (28) coincides with the main physical requirement determining the DM $\mathbf{P}$ and may be traced back to Dirac's equation for the time-independent Hamiltonian [19]. In turn, Eqs. (29) and (30) are additional system-structure-independent restrictions that follow from the idempotence requirement $\Pi^{2}=\Pi$ for the projector $\Pi=\frac{1}{2} \mathbf{P}$ and from the charge conservation condition, respectively.

Let the Hermitian matrix $\mathbf{Y}$ being sought be divided into four $n \times n$-dimensional submatrices as follows:

$$
\mathbf{Y}=\left|\begin{array}{cc}
\mathbf{V} & \mathbf{U} \\
\mathbf{U}^{+} & \mathbf{W}
\end{array}\right|
$$

Substituting Eq. (31) into Eq. (28) yields the following matrix equations:

$$
\begin{aligned}
& \mathbf{U B}^{+}=\mathbf{B U}^{+}, \quad \mathbf{U}^{+} \mathbf{B}=\mathbf{B}^{+} \mathbf{U}, \\
& \mathbf{V B}=\mathbf{B W}, \quad \mathbf{W B}^{+}=\mathbf{B}^{+} \mathbf{V} .
\end{aligned}
$$

Similarly, from Eq. (29) we obtain

$$
\begin{array}{cc}
\mathbf{V}^{2}+\mathbf{U U}^{+}=\mathbf{I}, & \mathbf{W}^{2}+\mathbf{U U}^{+}=\mathbf{I}, \\
\mathbf{V U}+\mathbf{U W}=\mathbf{0}, & \mathbf{U}^{+} \mathbf{V}+\mathbf{W U}^{+}=\mathbf{0} .
\end{array}
$$

It is seen that choice of zero diagonal blocks of the matrix $\mathbf{Y}$, i.e.,

$$
\mathbf{V}=\mathbf{W}=\mathbf{0}
$$

meets Eqs. (30) and (32)-(35). At the same time, Eqs. (32) and (34) yield the Hall conditions for the offdiagonal blocks $\mathbf{U}$ of the DM $\mathbf{P}$ and the relevant unitarity requirement, respectively. This implies a coincidence between matrices $\mathbf{U}$ and $\mathbf{Z}$ for this particular case, where $\mathbf{Z}$ is defined by Eqs. (13)-(15).

Thus, the Hall conditions follow directly from the commutation relation of Eq. (28), provided that Eq. (36) is accepted. The zero matrix requirements for blocks $\mathbf{V}$ and $\mathbf{W}$, in turn, prove to be equivalent to confinement to the charge-bond order matrix $\mathbf{P}$, corresponding to the ground state of our system, in which $n$ lowest-energy levels are occupied. Note that the noncanonical problem for the DM P represented by Eqs. (28)-(30) possesses numerous solutions corresponding to various ways of allocating the electrons in pairs to one-electron energy levels $[19,24]$.

To demonstrate the above-expected nature of Eq. (36), let us consider the stabilization energy of an alternant hydrocarbon, which may be alternatively defined as follows [19, 28]:

$$
\Delta E_{\text {stab }}=\operatorname{Trace}(\mathbf{H Y}) \text {. }
$$

Substituting the expressions for $\mathbf{Y}$ following from Eq. (27) and containing zero diagonal blocks into Eq. (37) yields the expression for $\Delta E_{\text {stab }}$ shown in Eq. (26) and corresponding to occupation of oneelectron states of the first eigenblock $\mathrm{E}_{1}$. The following additional remark should be made in this connection. Owing to zero diagonal blocks of the initial matrix $\mathbf{H}$ of Eq. (1), the submatrices $\mathbf{V}$ and $\mathbf{W}$ of the matrix $\mathrm{Y}$ do not contribute directly to the Trace of 
the product $\mathrm{HY}$, even if these are chosen to be nonzero matrices. In the latter case, however, the off-diagonal blocks $\mathbf{U}$ do not coincide with the matrix $\mathbf{Z}$, because the unitarity condition for the former $\left(\mathbf{U U}^{+}=\mathbf{I}\right)$ is then replaced by more involved conditions of Eq. (34).

It may be concluded that the same Hall conditions for off-diagonal blocks of the matrix being sought arise as intermediate steps of the solution of both the block-diagonalization problem and the commutation equation for the DM. This fact indicates a deep interrelation between the two fundamental noncanonical problems for alternant hydrocarbons and gives us an additional insight into the origin of similarity of matrices $\mathbf{C}$ and $\mathbf{P}$.

\section{Discussion of the Results Obtained}

Constitution of NCMOs of alternant hydrocarbons follows from Eqs. (19)-(21), (24) and (25). Coincidence of diagonal blocks of matrices $\mathbf{C}$ and $\mathbf{C}^{\prime}$ with unit matrices (I) ensures one-to-one correspondence between NCMOs and basis functions (AOs), as well as zero intrasubset delocalization of the former. Moreover, occupied and vacant NCMOs originate from different subsets of AOs.

Let the relative weights of basis functions within NCMOs be defined as squares of respective coefficients in linear combinations like that of Eqs. (20) and (25). It may then be concluded that each NCMO contains a principal basis orbital, the relative weight of which always coincides with $1 / 2$. Furthermore, any NCMO may be characterized by the set of $n$ partial delocalization coefficients $[14,15]$ defined in terms of relative weights of basis orbitals of the opposite subset. For example, the partial delocalization coefficient $\left(d_{(+) i,(2) k}\right)$ of the NCMO $\varphi_{(+) i}$ of Eq. (20) over the $k$ th $\mathrm{AO}$ of the second subset takes the form

$$
d_{(+) i,(2) k}=\frac{1}{2}\left[\left(\mathbf{B}^{+} \mathbf{R}\right)_{k i}\right]^{2}
$$

Moreover, the total delocalization coefficients of NCMOs $[14,15]$ may be defined, e.g.,

$$
D_{(+) i}=\sum_{k}^{(2)} d_{(+) i,(2) k}=\frac{1}{2} \sum_{k}^{(2)}\left[\left(\mathbf{B}^{+} \mathbf{R}\right)_{k i}\right]^{2} .
$$

From the normalization condition for NCMOs shown in Eq. (21), we then obtain

$$
D_{(+) i}=D_{(-) j}=\frac{1}{2},
$$

whatever the number of the NCMO. The same result also refers to NCMOs of Eq. (25).

Therefore, uniform total delocalization coefficients are peculiar to NCMOs of alternant hydrocarbons; these coincide with the relative weight of the principal AO. This also implies that partial delocalization coefficients of NCMOs cannot exceed the relative weight of the principal AO. Thus, NCMOs of alternant hydrocarbons are actually of the principal-orbital-and-tail constitution.

The final conclusion consists of the existence of common features of NCMOs of alternant hydrocarbons, as was the case with CMOs [21-23].

Let us turn now to the interrelation between NCMOs of alternant hydrocarbons and the respective charge-bond order matrix. Comparison of the relevant expressions of Eqs. (16), (23), and (27) shows that the vectors of coefficients of NCMOs coincide with the respective columns (rows) of the DM $\mathbf{P}$ (up to the normalization factor $1 / \sqrt{2}$ ). This implies that the shapes of particular NCMOs may be predicted on the basis of bond orders that are formed by the respective principal $\mathrm{AO}$ and the $\mathrm{AOs}$ of the opposite subset.

Substantial positive bond orders of conjugated hydrocarbons are known to arise for neighboring pairs of $2 p_{z}$ AOs corresponding to chemical bonds, while those for other (non-neighboring) pairs are at least two times smaller [20, 24, 29]. These trends in the relative values of bond orders allow us to expect that the shape of a specific $\operatorname{NCMO}\left(\varphi_{k}\right)$ depends decisively on the number of the nearest neighbors for the carbon atom $\left(\mathbf{C}_{k}\right)$, the respective principal $\mathrm{AO}\left(\chi_{k}\right)$ is pertinent to. In particular, two-center NCMOs of the bond-orbital-and-tail structure may be anticipated for the terminal carbon atoms of polyenes; three-center NCMOs are likely to be peculiar to atoms of cyclic hydrocarbons characterized by two neighbors; and so forth.

To illustrate the above general expectations, let us consider the occupied NCMOs of butadiene under an assumption about uniform resonance parameters for all neighboring pairs of $2 p_{z}$ AOs, viz. $\left(\chi_{1}, \chi_{2}\right),\left(\chi_{2}, \chi_{3}\right)$, and $\left(\chi_{3}, \chi_{4}\right)$. Bond orders of the terminal $\left(\mathrm{C}_{1}-\mathrm{C}_{2}\right.$ and $\left.\mathrm{C}_{3}-\mathrm{C}_{4}\right)$ and of the internal $\left(\mathrm{C}_{2}-\mathrm{C}_{3}\right)$ bonds are not uniform in this system [24, 29] in spite of the coincidence of resonance parameters, and we obtain 


$$
\begin{aligned}
& \varphi_{(+) 1}=\frac{1}{\sqrt{2}}\left(\chi_{1}+0.895 \chi_{2}-0.447 \chi_{4}\right), \\
& \varphi_{(+) 2}=\frac{1}{\sqrt{2}}\left(\chi_{3}+0.895 \chi_{4}+0.447 \chi_{2}\right) .
\end{aligned}
$$

The alternative set of occupied NCMOs following from Eq. (24), and attached to AOs $\chi_{2}$ and $\chi_{4}$, respectively, takes the form

$$
\begin{aligned}
& \varphi_{(+) 1}^{\prime}=\frac{1}{\sqrt{2}}\left(\chi_{2}+0.895 \chi_{1}+0.447 \chi_{3}\right), \\
& \varphi_{(+) 2}^{\prime}=\frac{1}{\sqrt{2}}\left(\chi_{4}+0.895 \chi_{3}-0.447 \chi_{1}\right) .
\end{aligned}
$$

NCMOs of Eqs. (41) and (42) closely resemble the bond orbitals of the terminal bonds. It should also be noted that analogous NCMOs were obtained for butadiene on the basis of solution of the blockdiagonalization problem in the form of power series [20].

The benzene molecule may be considered as another illustrative example. Three occupied NCMOs of similar structure will be obtained in this case, owing to the uniform constitution of the chain. For instance, the NCMO $\varphi_{(+) 1}$ attached to the AO $\chi_{1}$ takes the form

$$
\begin{aligned}
\varphi_{(+) 1} & =\frac{1}{\sqrt{2}}\left[\chi_{1}+\frac{2}{3}\left(\chi_{2}+\chi_{6}\right)-\frac{1}{3} \chi_{4}\right] \\
& =\frac{1}{\sqrt{2}}\left[\chi_{1}+0.667\left(\chi_{2}+\chi_{6}\right)-0.333 \chi_{4}\right]
\end{aligned}
$$

where $2 / 3=0.667$ coincides with the neighboring bond order of this molecule [29]. It is seen that the NCMO of Eq. (43) is a three-center orbital that involves an additional contribution of the $\mathrm{AO} \chi_{4}$, the latter taking the para-position with respect to the principal $\mathrm{AO} \chi_{1}$.

The structure of the $\mathrm{NCMO} \varphi_{(+) 1}$ reflects the well-known nonequivalence between meta- and ortho/para-positions, with respect to the given carbon atom $\mathrm{C}_{1}$ (cf. the weak and strong influences of a substituent upon meta- and ortho/para-positioned carbon atoms, respectively, in mono-substituted benzenes [5-7, 21, 22], as well as small and significant hyperfine splittings for the corresponding positions in the electron spin resonance (ESR) spectra of the benzyl radical [23]). Although the nonequivalence under discussion actually mani- fests itself only in substituent-containing systems, the result of Eq. (43) allows us to trace it back to the imminent peculiarities of electronic structures of the very benzene molecule.

Comparison of NCMOs of Eq. (43) to other types of noncanonical one-electron orbitals of benzene is also of interest. Thus, the translational-symmetrydetermined Wannier orbitals [30] may be found for the benzene-like ring of six AOs [31]. As with our $\mathrm{NCMOs}$, these may also be ascribed to separate $2 p_{z}$ AOs, so that the relevant coefficients take larger values as compared with the remaining ones. In contrast to NCMOs of Eq. (43), however, any Wannier orbital embraces all basis functions, and its signs alternate when passing from even- to oddnumbered AOs. The same features also refer to the Löwdin's natural orbitals of benzene (the so-called alternant orbitals) $[32,33]$ that are defined beyond the limits of the one-determinant approximation for the total wave function of $\pi$-electrons. These dissimilarities between our orbitals and those of Refs. [31-33] cause no surprise, as an unlimited number of different sets of NCMOs are known to exist for the benzene molecule [34].

Before completing this discussion, some possible fields of application of the results obtained may be mentioned. In general outline, these evidently coincide with respective fields for other types of localized orbitals [18]. The specific possibilities may be examplified by the use of NCMOs derived in this study as basis orbitals for separate fragments of molecules in the semilocalized approach to chemical reactivity [35]. Moreover, the NCMOs suggested along with the respective matrix $\mathbf{H}^{\prime}$ of Eqs. (2) and (18) are able to play the role of a zero order approximation in the noncommutative Rayleigh-Schrödinger perturbation theory $[16,28]$ aimed at dealing with block-diagonalization problems for matrices.

\section{Summary}

The contribution of the results obtained to the theory of electronic structures of molecules in general may be summarized as follows:

1. The results contribute to the development of direct ways of obtaining NCMOs of molecules without invoking CMOs and thereby provide us with an indirect support of equivalence of the localized and delocalized approaches to electronic structures.

2. The results demonstrate possibilities of the 
NCMO method in reflecting chemical structures of molecules and their alterations when passing from one class of compounds to another. Along with the well-known achievements of the $\mathrm{CMO}$ method in describing energy levels, these possibilities serve to substantiate the complementary nature of the two alternative methods of MOs [20].

3. The solution of the block-diagonalization problem for alternant hydrocarbons proposed in this article presents a new example of common quantum mechanical problems for entire classes of molecules [14, 15].

4. The solution suggested supplements the noncommutative Rayleigh-Schrödinger perturbation theory [16] with the case of two degenerated subsets of basis functions.

\section{References}

1. Zülicke, L. Quantenchemie B.1. Grundlagen and Algemeine Methoden; VEB Deutscher Verlag der Wissenschaften: Berlin, 1973.

2. McWeeny, R. Methods in Molecular Quantum Mechanics, 2nd ed.; Academic Press: London, 1992.

3. Huzinaga, S. The MO Method; Mir: Moscow, 1983 [in Russian].

4. Koopmans, T. A. Physica 1934, 1, 104.

5. Ingold, C. K. Structure and Mechanism in Organic Chemistry; Cornell University Press: Ithaca, NY, 1953.

6. March, J. Advanced Organic Chemistry, Reactions, Mechanisms and Structure; Wiley-Interscience: New York, 1985.

7. Hauptmann, S.; Gräfe, J.; Remane, H. Lehrbuch der Organischen Chemie; VEB Deutscher Verlag für Grundstoffindustrie: Leipzig, 1976.

8. Tedder, J. M.; Nechvatal, A. Pictorial Orbital Theory; Pitman: London, 1985.

9. Daudey, J. P. Chem Phys Lett 1974, 24, 574.
10. Mayer, I. Chem Phys Lett 1982, 89, 390.

11. Surjan, P. R.; Mayer, I.; Kertesz, M. J Chem Phys 1982, 77, 2454.

12. Mayer, I.; Surjan, P. R. J Chem Phys 1984, 80, 5649.

13. Gineityte, V. J Mol Struct (Theochem) 1993, 288, 111.

14. Gineityte, V. J Mol Struct (Theochem) 1995, 333, 297.

15. Gineityte, V. J Mol Struct (Theochem) 1995, 343, 183.

16. Gineityte, V. Int J Quantum Chem 1998, 58, 119.

17. Gineityte, V. Int J Quantum Chem 1999, 72, 559.

18. Chalvet, O. (Ed.); Localization and Delocalization in Quantum Chemistry, Atoms and Molecules in the Ground State, Vol. 1, Reidel: Dordrecht, 1975.

19. Mestechkin, M. M. Metod Matricy Plotnosti v Teorii Molekul, Naukova Dumka: Kiev, 1977 [in Russian].

20. Gineityte, V. J Mol Struct (Theochem) 1999, 487, 231.

21. Dewar, M. J. S. The Molecular Orbital Theory of Organic Chemistry; McGraw-Hill: New York, 1969.

22. Dewar, M. J. S.; Dougherty, R. C. The PMO Theory of Organic Chemistry; Plenum: New York, 1975.

23. Segal, G. A. (Ed.); Semiempirical Methods of Electronic Structure Calculations; Part A: Techniques; Plenum: New York, 1977.

24. Hall, G. G. Proc R Soc (Lond) 1955, A229, 251.

25. Horn, R. A.; Johnson, Ch. R. Matrix Analysis; Cambridge University Press: Cambridge, 1986.

26. Korn, G. A.; Korn, T. M. Mathematical Handbook for Scientists and Engineers; McGraw-Hill: New York, 1961.

27. Potts, R. B. J Chem Phys 1953, 21, 758.

28. Gineityte, V. Int J Quantum Chem 2000, 77, 534.

29. Basilevskii, M. V. Metod Molekuliarnych Orbit i Reaktsionnaya Sposobnost Organitcheskich Molekul; Khimia: Moscow, 1969 [in Russian].

30. Wannier, G. H. Phys Rev 1937, 52, 191.

31. Slater, J. C. Electronic Structure of Molecules; McGraw-Hill: New York, 1963.

32. Löwdin, P. O. Phys Rev 1955, 97, 1474, 1490, 1509.

33. Itoh, T.; Yoshizumi, H. J Phys Soc Jpn 1955, 10, 201.

34. Edmiston, C.; Rudenberg, K. In Quantum Theory of Atoms, Molecules and the Solid State; Löwdin, P. O. (Ed.); Academic Press: New York, 1966; p 263.

35. Gineityte, V. Int J Quantum Chem 2003, 94, 302. 